\title{
Sensory Processing Problems in Children with ADHD, a Systematic Review
}

\author{
Ahmad Ghanizadeh ${ }^{1,2} \bowtie$ \\ ${ }^{1}$ Research Center for Psychiatry and Behavioral Sciences, ${ }^{2}$ Department of Psychiatry, Shiraz University of Medical Sciences, Hafez Hospital, \\ Shiraz, Iran
}

One of the most common psychiatric disorders in children is attention deficit hyperactivity disorder (ADHD). Its course and outcome are heterogeneous. Sensory processing problems impact the nature of response to daily events. ADHD and sensory problems may occur together and interact. No published review article about sensory processing problems in children with ADHD were found. A systematic search, conducted on Pub-Med (up to January 2010), and Google Scholar, yielded 255 abstracts on sensory processing problems in children including 11 studies about sensory problems in children with ADHD. Sensory processing problems in children with ADHD is not a well studied area. Sensory processing problems in children with ADHD are more common than in typically developing children. Findings do not support that ADHD subtypes are distinct disorders with regard to sensory processing problems. However, co-morbidity with oppositional defiant disorder and anxiety are predictors of more severe sensory processing problems in children with ADHD.

Psychiatry Investig 2011;8:89-94

Key Words Sensory processing problems, ADHD, Review.

\section{INTRODUCTION}

\section{Attention deficit hyperactivity disorder}

The symptoms of attention deficit hyperactivity disorder (ADHD) are relatively common in the community sample of children. The rates for boys and girls are $13.6 \%$ and $6.5 \%$, respectively. ${ }^{1}$ This disorder is usually co-morbid with other disorders. More than two thirds of the clinical samples of children with ADHD have at least one co-morbid psychiatric disorder which are more likely to be oppositional defiant and anxiety disorders. This high rate of psychiatric disorders is not just confined to the children but their families also report a high rate of psychiatric disorders. ${ }^{2,3}$ The rate of psychiatric disorder co-morbidities does not differentiate between ADHD subtypes. ${ }^{4}$

Received: June 4, 2010 Accepted: September 20, 2010

Available online: November 20, 2010

$\square$ Correspondence: Ahmad Ghanizadeh, MD

Research Center for Psychiatry and Behavioral Sciences, Department of Psychiatry, Shiraz University of Medical Sciences, Hafez Hospital,

Shiraz, Iran

Tel: +98-711-627-30-70, Fax: +98-711-627-30-70

E-mail: ghanizad@sina.tums.ac.ir

(a) This is an Open Access article distributed under the terms of the Creative Commons Attribution Non-Commercial License (http://creativecommons.org/licenses/bync/3.0) which permits unrestricted non-commercial use, distribution, and reproduction in any medium, provided the original work is properly cited.

\section{Sensory processing problems}

Sensory processing disorders are impairments in responding to sensory stimuli such as impairments in detection, modulation, or interpretation of stimuli. ${ }^{5}$ Sensory processing problems impact the responses of children to sensory events in daily life. ${ }^{6}$ $5.3 \%$ of the kindergarten children meet screening criteria for sensory processing disorders according to their parental reports. ${ }^{7}$ One type of sensory processing problem is sensory overresponsivity or sensory hypersensitivity. That is, individuals respond to sensory stimuli in the way that is faster, longer, or more intense than what is expected. ${ }^{8}$ This response can be towards any types of sensory stimuli. Sensory over-responsivity can be considered as an independent diagnosis. ${ }^{9}$ For example, a child with tactile sensitivity or defensiveness might be defensive for hair-brushing and/or haircuts because she/he cannot tolerate it easily.

Another form of sensory processing problems is under-responsivity. Individuals with under-responsivity are unaware or they are slow to respond to sensory input. The third type is sensory-seeking, where individuals crave or display interest in sensory experiences. ${ }^{10}$ Sensory discrimination problems are another type of sensory processing problem characterized by difficulty interpreting the specific characteristics of sensory stimuli. ${ }^{11}$ There are multiple sensory modalities but they are not separate 
and interact with each other. For example, sound alters vision or vision alters sound location. ${ }^{12}$ An example is that when we watch television or movies, we perceive that the voices originate from the actors on the screen while there is a large spatial discrepancy between the origine of sound and the actors. ${ }^{12}$

The etiology of sensory processing is unknown. Genetic and familial factors have been suggested. ${ }^{11}$ Others suggest other risk factors such as pre-, peri- and post-natal birth factors such as maternal stress during pregnancy, jaundice, and allergies. ${ }^{13}$

It is clear that there is an alteration in the neural networks and a possible central role of dopamine for sensory problems that cannot be linked to specific cerebral lesions in children with ADHD. ${ }^{14}$ One of the prominent neuroanatomical markers for children and adolescents with ADHD is widespread cortical thickness reductions. ${ }^{15}$ Activities in resting state in sensory and sensory-related cortices in ADHD is significantly more than those of the control group. ${ }^{16}$ Moreover, there is a connection between perception and action that is impaired in $\mathrm{ADHD} .{ }^{17}$ There are growing published studies about sensory processing problems in children with ADHD. However, the underlying structure and pathophysiology is not well studied and the assessment of sensory profiles of children with behavioral problems in clinical practice is recommended. ${ }^{18}$

The reason for conducting this systematic review is that there are some studies about sensory processing problems in children with ADHD but there appears to be no current reviews on sensory processing problems in ADHD.

\section{METHODS}

The MEDLINE database was systemically searched for articles published up to January 2010 reporting sensory processing problems in children with $\mathrm{ADHD}$. The keywords used were $\mathrm{ADHD}$, attention deficit hyperactivity disorder, sensory processing problem, sensory processing modalities, sensory processing integration, sensory, reactivity, auditory, tactile, vestibular, olfactory, visual, and profile. The reference section of the identified articles was reviewed. Google scholar database was searched for possible related articles. All of the articles were in English. There was not found any review article about sensory processing problems in children with ADHD. Two hundreds fity five abstracts were retrieved and studied. Eleven articles about sensory problems in children with $\mathrm{ADHD}$ were found (Table 1).

\section{RESULTS}

Most of the studies were published in recent years. They usually included small samples of children with ADHD (for example, ${ }^{19,20}$ All of the studies used questionnaire or parent-reported measures. More details about the year of publication, sample size, the age of sample, and the measures used for assessment are displayed I Table 1.

The sensory-motor abilities of ADHD children are less than those of control group. ${ }^{21}$ Sensory processing problems in children with ADHD are more common than children without $\mathrm{ADHD}^{22}$ and the patterns of sensory modulation in the ADHD

Table 1. Characteristics of studies

\begin{tabular}{|c|c|c|c|c|}
\hline Authors & Year & Sample size (ADHD) & Mean of age (SD) or range & Measure \\
\hline Parush et al. & 2007 & 67 , only boys & $5-11$ years & $\begin{array}{l}\text { Touch inventory for preschoolers, the sensory } \\
\text { reactivity score, somatosensory evoked potential, } \\
\text { sensory integration and praxis test }\end{array}$ \\
\hline Bröring et al. & 2008 & 47 & Mean age 9 y 8 mo [SD 1 y 11 mo] & $\begin{array}{l}\text { Touch inventory for } \\
\text { elementary-school-aged children }\end{array}$ \\
\hline Yochman et al. & 2006 & 49 & 4 y 7 mo [SD 7 mo]; range 3 y 10 mo- 6 y & The sensory profile \\
\hline Mangeot et al. & 2001 & 26 & Mean age 8.3 years & Short sensory profile \\
\hline Reynolds et al. & 2009 & 24 & $6-10$ & Sensory over-responsivity inventory \\
\hline Ermer et al. & 2001 & 61 & $3-15$ & Sensory profile \\
\hline Cheung et al. & 2009 & 114 & $4.8-12$ years $(\mathrm{M}=7.9$ years, $\mathrm{SD}=1.4)$ & Sensory profile \\
\hline Iwanaga & 2006 & 45 , only boys & 45 to 72 months & Miller assessment for preschoolers \\
\hline Yochman et al. & 2004 & 48 & $4-6$ & Sensory profile questionnaire \\
\hline Ghanizadeh & 2008 & 81, clinical & $8.4(1.9)$ & $\begin{array}{l}\text { Tactile sensory dysfunction including } \\
\text { hypersensitivity, hyposensitivity, and poor tactile } \\
\text { perception and discrimination (PTPD) }\end{array}$ \\
\hline Ghanizadeh & 2009 & 104, clinical & $8.5(1.8)$ & $\begin{array}{l}\text { Auditory processing problem checklist including } \\
\text { hypersensitivity to sounds (HES)(or auditory } \\
\text { defensiveness) and hyposensitivity to sound (HOS) }\end{array}$ \\
\hline
\end{tabular}

ADHD: attention deficit hyperactivity disorder 
are significantly different from typical children. ${ }^{23}$

The sensory profile of children can discriminate children with autism or pervasive developmental disorder (PDD), children with ADHD, and children without disabilities. ${ }^{22,24}$

The sensory processing problem in ADHD is reported in both of the physiological and parent-reported measures. ${ }^{19}$ The sensory processing problem is not gender related but it is associated with age. 22

Specific sensory symptoms are correlated with particular behavioral problems such as aggression and delinquency in ADHD. The higher level of sensory problems in ADHD predicts the higher levels of aggression and delinquency. ${ }^{19}$ The impairment of receiving and processing of sensory information in children with ADHD may cause inappropriate responses at different settings such as school, home and in the community. ${ }^{25}$

In addition, sensory functioning has a strong correlation with academic achievement and cognitive processing in ADHD. ${ }^{26}$ Earlier detection and management of sensory processing problems can play an important role for the performance improvement in children with ADHD. The early assessment of sensory-motor skills is suggested to be used for the prediction of academic performance deficits. ${ }^{26}$

\section{DISCUSSION}

\section{Tactile sensory}

Children with ADHD have more difficulties in tactile processing. ${ }^{27}$ The level of tactile defensiveness in females with ADHD is higher than that of males with ADHD. Tactile defensiveness is not part of a familial risk for ADHD because it is specific to children with ADHD and not to their sibling without ADHD. ${ }^{28}$ Tactile defensiveness is not due to tactile perception impairment but the impairment of central processing of somatosensory information. ${ }^{29}$

Sensory over-responsivity in ADHD is associated with anxiety. These children have a higher level of anxiety than those ADHD children without sensory overresponsivity and nonADHD children. ${ }^{20}$ The hypothalamic pituitary adrenal axis is influenced by tactile sensory overresponsivity in children with ADHD. ${ }^{30}$ The symptoms of oppositional defiant disorder are predictors in hypersensitivity scores of tactile sensory function in ADHD. This impairment is not related to ADHD subtypes or gender. ${ }^{31}$ Another study reported that tactile defensiveness in girls with $\mathrm{ADHD}$ is more than boys with $\mathrm{ADHD}$ and the level of tactile defensiveness in boys was not different from the control boys. ${ }^{28}$ Two samples of tactile defensiveness are "doesn't like to brush his/her hair or is easily annoyed by it" and "react excessively to small cuts, or bites". "Might not be aware that his/ her face or hands are dirty or even his/her runny nose" and "may not be aware that he/she has been touched, except that when he/ she has been touched forcefully" are two examples for hyposensitivity to touch.

\section{Balance control and vestibular systems}

There are very few reports about balance problems in $\mathrm{AD}$ HD. ${ }^{32}$ Balance performance ${ }^{33}$ and ability for equilibrium and postural control ${ }^{21}$ in children with ADHD combined type are more impaired in comparison to typically developing children. More than one third of children with ADHD have poor balance and coordination. ${ }^{34}$ This balance problem in ADHD is associated with the sensory inputs, the sensory integration, and/ or the inhibition of excessive movements. ${ }^{35}$ Children with combined-type ADHD without learning disorder do not usually benefit from vestibular stimulation. ${ }^{36}$ More studies considering covariant factors are required to reach a firm conclusion. ${ }^{37}$

\section{Auditory sensory}

Individuals with auditory processing disorders have normal peripheral hearing but they may be unsure about what they hear. ${ }^{38}$ Difficulty in auditory discrimination or localization and distractibility are other forms of auditory processing disorders. ${ }^{39}$ Hyposensitivity to sounds or under-registering of sounds can motivate parents seek professional help for their inattentive children. It may seem like a learning disability. Two examples of hypersensitivity to sounds are: "Often pleased by asking people to keep quiet" and "Very sensitive towards sounds which are unheard by others such as humming of refrigerator, clocks ticking, or fans". Two examples of hyposensitivity to sounds are "often irresponsive to oral points or calls" and "seems puzzled about where origin of a sound is". Auditory processing problem in children with ADHD is an ignored research area. ${ }^{40}$ The auditory processing of children without any disability is better than that in children with ADHD. ${ }^{22}$ Of course, this difficulty is not specific to ADHD and it cannot differentiate children with $\mathrm{AD}$ HD and those with autism. ${ }^{22}$

The subtypes of ADHD are not different regarding auditory processing problems. However, the co-morbidity of ODD in children with ADHD is a risk factor for auditory processing problems. ${ }^{40}$ These children are more under-responsive to sounds. Background noise in their classroom should be minimized. Co-morbidity with anxiety predicts hypersensitivity to the sound. Impairment of auditory processing is not gender-related in children with $\mathrm{ADHD} .{ }^{40}$ Considering the co-occurrence of ODD and auditory processing problem, the question is that they are distinct disorders or they are part of a broader problem.

\section{Visual and olfactory function}

Stimulants may induce visual oversensitivity or photophobia in children with ADHD. ${ }^{41}$ Stimulants impact the visual fields in children with $\mathrm{ADHD}{ }^{42}$ There are only three studies concern- 
ing association of olfactory function in children with ADHD. Odor threshold is lower in ADHD than in healthy control subjects and stimulants improve it. However, odor discrimination or identification is not different in ADHD in comparison to the control group. ${ }^{43}$ Another study reported that the olfactory identification ability of children with ADHD is not as good as healthy controls. ${ }^{44}$ Prefrontal compromise is suggested as a reason for this inability and ADHD symptoms. ${ }^{44}$ Stimulants improve the odor sensitivity in ADHD while odor discrimination and identification are not different between ADHD children and control group. ${ }^{43}$

Sensory processing problems may differentiate ADHD from normally developing children. However, it does not mean that it is specific to ADHD. The sensory profiles of children with ADHD may be similar to other disabilities such as autism. ${ }^{22}$ Thus, how do we differentiate the different disorders in terms of sensory processing problems? Other assessment characteristics would be required to show up the differences in clinical features between the two groups.

Sensory problems are usually assessed using parents or caregivers reported questionnaires. The Sensory Profile is a widely used parent reported standardized questionnaire. Children's responses to different sensory events in daily life are rated by their parents. It has been used in different samples such as children with $\mathrm{ADHD}^{25}$ Auditory processing, visual processing, taste/smell processing, body position, movement, touch processing, activity level, and social/emotional responses are the dimensions assessed by this questionnaire.

The parent reported auditory processing problem checklist which is used for assessment of auditory processing problems indicates child's reaction to sounds. Its psychometric properties were reported before. ${ }^{40}$ The two aspects of auditory dysfunction are hypersensitivity to sounds (or auditory defensiveness) and hyposensitivity to sound (under-registers) ${ }^{40}$

Tactile sensory dysfunction checklist is an instrument used for measuring tactile dysfunction. It comprises three types of tactile dysfunction including hypersensitivity to touch (tactile defensiveness), hyposensitivity to touch (under-responsive), and poor tactile perception and discrimination. ${ }^{31}$

ADHD children with sensory oversensitivity usually suffer from other problems such as anxiety. ${ }^{20}$ Treatment plans for children with ADHD and sensory oversensitivity should include management of other co-morbid psychiatric problems. As parents of children are educated about ADHD symptoms, ${ }^{45}$ considering the co-morbidity of ADHD and sensory processing problems, they should also be educated about the symptoms and behavioral management of sensory processing problems. ${ }^{46}$ In a promising report, it is indicated that occupational therapy improves sensory processing problems. ${ }^{5}$ Moreover, there are some reports showing that sensory processing problems can guide clinicians for pharmacological management of children with $\mathrm{ADHD}^{47}$ or other disorders. ${ }^{48}$ Although, there are some speculation that some medications may improve sensory problems, ${ }^{49}$ it is not clear how much can pharmacotherapy improve sensory problems in ADHD. An area that needs to be researched is effectiveness of interventions.

\section{Future studies}

Further studies may be conducted with objective measures such as Somatosensory Evoked Potential, ${ }^{29}$ P300 auditory evoked potentials, ${ }^{50}$ digital optical motion capture instruments, ${ }^{32}$ electrodermal reactivity, and Pennsylvania Smell Identification Test. ${ }^{44}$ Providing more objective measures and laboratory findings improves differentiating sensory processing problems from other disorders or problems. Moreover, there are some reports about the role of genetics on the sensory gating of mid-latency auditory evoked responses. ${ }^{51}$ So, future studies should investigate the possible role of genetics factors on the sensory processing problems in children with ADHD.

Overall, considering the fact that sensory problems in children with ADHD can impact the behavior and education of these children, it needs to be focused in future studies because it is not widely studied. More developed, valid, and reliable measurements are required. Many of the published studies have only focused on one or few sensory processing types. Future studies should include investigation of different sensory modalities. Since ADHD is not a homogenous disorder and previous studies have usually included just one type of $\mathrm{AD}$ $\mathrm{HD}$, it is recommended that further studies include different types of ADHD and also consider psychiatric co-morbidities in children. Probably, ADHD children tend to have more than one type of sensory processing problems. It can guide clinicians for a better classification of ADHD according to the types of problem, severity, and duration. Our knowledge about association of sensory problems and age, gender, socioeconomic status of family, co-occurrence with medical and psychological conditions, parent-child interaction, mental age, severity of $\mathrm{AD}$ $\mathrm{HD}$, and environmental factors such as environmental noise needs to be increased. Studies including measurement of possible biomarkers such as brain neurotransmitters, structural and functional aspects of sensory processing problems should be investigated.

Most of the published articles regarding sensory problems relay on parent reports, considering that parents of these children usually suffer from psychiatric disorders such as depression and anxiety. It is not studied if parental psychiatric problems may impact on their report for children's sensory processing problem. Further studies may investigate if the sensory processing problems are behavioral biomarkers for $\mathrm{ADHD}$. In other words, both basic and clinical sciences about it need more 
studies. Research in this field may help to re-organize our understanding from ADHD and its diagnosis. Possible children and maternal risk factors for sensory problems need to be studied.

\section{CONCLUSION}

Sensory processing problems in children with ADHD is not a well studied area. There are many reported limitations for the published studies. So, it is difficult to reach a firm conclusion due to the limitations. However, it is reported that the sensory processing problems are more common in children with $\mathrm{ADHD}$ than in typically developing children. This article emphasizes that sensory processing problem in ADHD need to be further studied. Treatment protocols for management of this problem should be provided and researched.

\section{REFERENCES}

1. Ghanizadeh A. Distribution of symptoms of attention deficit-hyperactivity disorder in schoolchildren of Shiraz, south of Iran. Arch Iran Med 2008;11:618-624.

2. Ghanizadeh A, Mohammadi MR, Moini R. Comorbidity of psychiatric disorders and parental psychiatric disorders in a sample of Iranian children with ADHD. J Atten Disord 2008;12:149-155.

3. Byun H, Yang J, Lee M, Jang W, Yang JW, Kim JH, et al. Psychiatric comorbidity in Korean children and adolescents with attention-deficit hyperactivity disorder: psychopathology according to subtype. Yonsei Med J 2006;47:113-121.

4. Ghanizadeh A. Psychiatric comorbidity differences in clinic-referred children and adolescents with ADHD according to the subtypes and gender. J Child Neurol 2009;24:679-684.

5. Miller LJ, Coll JR, Schoen SA. A randomized controlled pilot study of the effectiveness of occupational therapy for children with sensory modulation disorder. Am J Occup Ther 2007;61:228-238.

6. Yochman A, Parush S, Ornoy A. Responses of preschool children with and without ADHD to sensory events in daily life. Am J Occup Ther 2004; 58:294-302.

7. Ahn RR, Miller LJ, Milberger S, McIntosh DN. Prevalence of parents' perceptions of sensory processing disorders among kindergarten children. Am J Occup Ther 2004;58:287-293.

8. Miller LJ, Anzalone ME, Lane SJ, Cermak SA, Osten ET. Concept evolution in sensory integration: a proposed nosology for diagnosis. Am J Occup Ther 2007;61:135-140.

9. Reynolds S, Lane SJ. Diagnostic validity of sensory over-responsivity: a review of the literature and case reports. J Autism Dev Disord 2008;38: 516-529.

10. Ben-Sasson A, Cermak SA, Orsmond GI, Tager-Flusberg H, Kadlec $\mathrm{MB}$, Carter AS. Sensory clusters of toddlers with autism spectrum disorders: differences in affective symptoms. J Child Psychol Psychiatry 2008; 49:817-825.

11. Miller LJ, Nielsen DM, Schoen SA, Brett-Green BA. Perspectives on sensory processing disorder: a call for translational research. Front Integr Neurosci 2009;3:22.

12. Shimojo S, Shams L. Sensory modalities are not separate modalities: plasticity and interactions. Curr Opin Neurobiol 2001;11:505-509.

13. May-Benson TA, Koomar JA, Teasdale A. Incidence of pre-, peri-, and post-natal birth and developmental problems of children with sensory processing disorder and children with autism spectrum disorder. Front Integr Neurosci 2009;3:31.
14. Pasini A, D’agati E. Pathophysiology of NSS in ADHD. World J Biol Psychiatry 2009;10:495-502.

15. Narr KL, Woods RP, Lin J, Kim J, Phillips OR, Del'homme M, et al. Widespread cortical thinning is a robust anatomical marker for attentiondeficit/hyperactivity disorder. J Am Acad Child Adolesc Psychiatry 2009; 48:1014-1022.

16. Tian L, Jiang T, Liang M, Zang Y, He Y, Sui M, et al. Enhanced restingstate brain activities in ADHD patients: a fMRI study. Brain Dev 2008; 30:342-348.

17. Dockstader C, Gaetz W, Cheyne D, Tannock R. Abnormal neural reactivity to unpredictable sensory events in attention-deficit/hyperactivity disorder. Biol Psychiatry 2009;66:376-383.

18. Shochat T, Tzischinsky O, Engel-Yeger B. Sensory hypersensitivity as a contributing factor in the relation between sleep and behavioral disorders in normal schoolchildren. Behav Sleep Med 2009;7:53-62.

19. Mangeot SD, Miller LJ, McIntosh DN, McGrath-Clarke J, Simon J, Hagerman RJ, et al. Sensory modulation dysfunction in children with attention-deficit-hyperactivity disorder. Dev Med Child Neurol 2001; 43:399-406.

20. Reynolds S, Lane SJ. Sensory overresponsivity and anxiety in children with ADHD. Am J Occup Ther 2009;63:433-440.

21. Iwanaga R, Ozawa H, Kawasaki C, Tsuchida R. Characteristics of the sensory-motor, verbal and cognitive abilities of preschool boys with attention deficit/hyperactivity disorder combined type. Psychiatry Clin Neurosci 2006;60:37-45.

22. Cheung PP, Siu AM. A comparison of patterns of sensory processing in children with and without developmental disabilities. Res Dev Disabil 2009;30:1468-1480.

23. Yochman A, Ornoy A, Parush S. Co-occurrence of developmental delays among preschool children with attention-deficit-hyperactivity disorder. Dev Med Child Neurol 2006;48:483-488.

24. Ermer J, Dunn W. The sensory profile: a discriminant analysis of children with and without disabilities. Am J Occup Ther 1998;52:283-290.

25. Dunn W, Bennett D. Patterns of sensory processing in children with attention deficit hyperactivity disorder. Occup Ther J Res 2002;22:4-15.

26. Davis AS, Pass LA, Finch WH, Dean RS, Woodcock RW. The canonical relationship between sensory-motor functioning and cognitive processing in children with attention-deficit/hyperactivity disorder. Arch Clin Neuropsychol 2009;24:273-286.

27. Hern KL, Hynd GW. Clinical differentiation of the attention deficit disorder subtypes: do sensorimotor deficits characterize children with ADD/ WO? Arch Clin Neuropsychol 1992;7:77-83.

28. Bröring T, Rommelse N, Sergeant J, Scherder E. Sex differences in tactile defensiveness in children with ADHD and their siblings. Dev Med Child Neurol 2008;50:129-133.

29. Parush S, Sohmer H, Steinberg A, Kaitz M. Somatosensory function in boys with ADHD and tactile defensiveness. Physiol Behav 2007;90:553558.

30. Reynolds S, Lane SJ, Gennings C. The moderating role of sensory Overresponsivity in HPA activity: a pilot study with children diagnosed with ADHD. J Atten Disord 2009;13:468-478.

31. Ghanizadeh A. Tactile sensory dysfunction in children with ADHD. Behav Neurol 2008;20:107-112.

32. Rochelle KS, Witton C, Talcott JB. Symptoms of hyperactivity and inattention can mediate deficits of postural stability in developmental dyslexia. Exp Brain Res 2009;192:627-633.

33. Shum SB, Pang MY. Children with attention deficit hyperactivity disorder have impaired balance function: involvement of somatosensory, visual, and vestibular systems. J Pediatr 2009;155:245-249.

34. Sergeant JA, Piek JP, Oosterlaan J. ADHD and DCD: a relationship in need of research. Hum Mov Sci 2006;25:76-89.

35. Zang Y, Gu B, Qian Q, Wang Y. Objective measurement of the balance dysfunction in attention deficit hyperactivity disorder children. Chin J Clin Rehabil 2002;6:1372-1374.

36. Clark DL, Arnold LE, Crowl L, Bozzolo H, Peruggia M, Ramadan Y, et 
al. Vestibular Stimulation for ADHD: randomized controlled trial of Comprehensive Motion Apparatus. J Atten Disord 2008;11:599-611.

37. Ghanizadeh A. Commentary to: Postural and gait performance in children with attention deficit/hyperactivity disorder [Gait Posture 2008]. Gait Posture 2009;29:661; author reply 662.

38. Jerger J, Musiek F. Report of the Consensus Conference on the Diagnosis of Auditory Processing Disorders in School-Aged Children. J Am Acad Audiol 2000;11:467-474.

39. Bamiou DE, Musiek FE, Luxon LM. Aetiology and clinical presentations of auditory processing disorders--a review. Arch Dis Child 2001; 85:361-365

40. Ghanizadeh A. Screening signs of auditory processing problem: does it distinguish attention deficit hyperactivity disorder subtypes in a clinical sample of children? Int J Pediatr Otorhinolaryngol 2009;73:81-87.

41. Ghanizadeh A, Aghakhani K. Photophobia and methylphenidate. Psychopharmacol Bull 2008;41:171-173.

42. Ghanizadeh A. Visual fields in children with attention-deficit/hyperactivity disorder before and after treatment with stimulants. Acta Ophthalmol 2010;88:e56.

43. Romanos M, Renner TJ, Schecklmann M, Hummel B, Roos M, von Mering C, et al. Improved odor sensitivity in attention-deficit/hyperactivity disorder. Biol Psychiatry 2008;64:938-940.

44. Karsz FR, Vance A, Anderson VA, Brann PG, Wood SJ, Pantelis C, et al. Olfactory impairments in child attention-deficit/hyperactivity disorder. J Clin Psychiatry 2008;69:1462-1468.

45. Ghanizadeh A. Educating and counseling of parents of children with attention-deficit hyperactivity disorder. Patient Educ Couns 2007;68: 23-28.

46. Cheng M, Boggett-Carsjens J. Consider sensory processing disorders in the explosive child: case report and review. Can Child Adolesc Psychiatr Rev 2005;14:44-48.

47. Ghanizadeh A. Can behavioral sensory processing problems guide us to a better pharmacological management of children with attention deficit hyperactivity disorder?: a case report. Psychiatry (Edgmont) 2009;6: 40-43.

48. Ghanizadeh A. Should tactile sensation impairment be considered in pharmacotherapy of pervasive developmental disorders? A case report. Neuro Endocrinol Lett 2008;29:877-878

49. Ghanizadeh A. Does risperidone improve hyperacusia in children with autism? Psychopharmacol Bull 2009;42:108-110.

50. Schochat E, Scheuer CI, Andrade ER. ABR and auditory P300 findings in children with ADHD. Arq Neuropsiquiatr 2002;60:742-747.

51. Anokhin AP, Vedeniapin AB, Heath AC, Korzyukov O, Boutros NN. Genetic and environmental influences on sensory gating of mid-latency auditory evoked responses: a twin study. Schizophr Res 2007;89:312319 . 